\title{
Some Convergence and Stability Results for Two New Kirk Type Hybrid Fixed Point Iterative Algorithms
}

\author{
Faik Gürsoy ${ }^{1}$ and Vatan Karakaya ${ }^{2}$ \\ ${ }^{1}$ Department of Mathematics, Yildiz Technical University, Davutpasa Campus, Esenler, 34220 Istanbul, Turkey \\ ${ }^{2}$ Department of Mathematical Engineering, Yıldız Technical University, Davutpasa Campus, Esenler, 34210 Istanbul, Turkey
}

Correspondence should be addressed to Faik Gürsoy; faikgursoy02@hotmail.com

Received 13 September 2013; Accepted 13 November 2013; Published 28 January 2014

Academic Editor: M. Mursaleen

Copyright (C) 2014 F. Gürsoy and V. Karakaya. This is an open access article distributed under the Creative Commons Attribution License, which permits unrestricted use, distribution, and reproduction in any medium, provided the original work is properly cited.

We introduce Kirk-multistep-SP and Kirk-S iterative algorithms and we prove some convergence and stability results for these iterative algorithms. Since these iterative algorithms are more general than some other iterative algorithms in the existing literature, our results generalize and unify some other results in the literature.

\section{Introduction and Preliminaries}

Fixed point theory has an important role in the study of nonlinear phenomena. This theory has been applied in a wide range of disciplines in various areas such as science, technology, and economics; see, for example, [1-5]. The importance of this theory has attracted researchers' interest, and consequently numerous fixed point theorems have been put forward; see, for example, [6-17] and the references included therein. In this highly dynamic area, one of the most celebrated theorems amongst hundreds is Banach fixed point theorem (also known as the contraction mapping theorem or contraction mapping principle) [7]. An important process which is called iteration method arises naturally during proving of this theorem. A fixed point iteration method is given by a general form as follows:

$$
\begin{gathered}
x_{0} \in X, \\
x_{n+1}=f\left(T, x_{n}\right), \quad \forall n \in \mathbb{N},
\end{gathered}
$$

where $X$ is an ambient space, $x_{0}$ is an arbitrary initial point, $T: X \rightarrow X$ is an operator, and $f$ is some function. For example, if $f\left(T, x_{n}\right)=T x_{n}$ in (1), then we obtain well-known Picard iteration [18] as follows:

$$
\begin{gathered}
x_{0} \in X, \\
x_{n+1}=f\left(T, x_{n}\right)=T x_{n}, \quad \forall n \in \mathbb{N} .
\end{gathered}
$$

Iterative methods are important instruments commonly used in the study of fixed point theory. These powerful and useful tools enable us to find solutions for a wide variety of problems that arise in many branches of the above mentioned areas. This is a reason, among a number of reasons, why researchers are seeking new iteration methods or trying to improve existing methods over the years. In this respect, it is not surprising to see a number of papers dealing with the study of iterative methods to investigate various important themes; see, for example, [19-27].

The purpose of this paper is to introduce two new Kirk type hybrid iteration methods and to show that these iterative methods can be used to approximate fixed points of certain class of contractive operators. Furthermore, we prove that these iterative methods are stable with respect to the same class of contractive operators.

As a background to our exposition, we describe some iteration schemes and contractive type mappings.

The following multistep-SP iteration was employed in [20, 28]:

$$
\begin{gathered}
x_{0} \in X, \\
x_{n+1}=\left(1-\alpha_{n}\right) y_{n}^{1}+\alpha_{n} T y_{n}^{1}, \\
y_{n}^{i}=\left(1-\beta_{n}^{i}\right) y_{n}^{i+1}+\beta_{n}^{i} T y_{n}^{i+1}, \\
y_{n}^{k-1}=\left(1-\beta_{n}^{k-1}\right) x_{n}+\beta_{n}^{k-1} T x_{n}, \quad n \in \mathbb{N},
\end{gathered}
$$


where $\mathbb{N}$ denotes the set of all nonnegative integers, including zero, and $\left\{\alpha_{n}\right\}_{n=0}^{\infty},\left\{\beta_{n}\right\}_{n=0}^{\infty},\left\{\gamma_{n}\right\}_{n=0}^{\infty}$, and $\left\{\beta_{n}^{i}\right\}_{n=0}^{\infty}, i=\overline{1, k-2}$, $k \geq 2$, are real sequences in $[0,1)$ satisfying certain conditions.

By taking $k=3$ and $k=2$ in (3) we obtain SP [25] and two-step Mann [27] iterative schemes, respectively. In (3), if we take $k=2$ with $\beta_{n}^{1}=0$ and $k=2$ with $\beta_{n}^{1} \equiv 0, \alpha_{n} \equiv$ $\lambda$ (const.), then we get the iterative procedures introduced in $[23,29]$, which are commonly known as the Mann and Krasnoselskij iterations, respectively. The Krasnoselskij iteration [29] reduces to the Picard iteration [18] for $\lambda=1$.

A sequence $\left\{x_{n}\right\}_{n=0}^{\infty}$ defined by

$$
\begin{gathered}
x_{0} \in X, \\
x_{n+1}=\left(1-\alpha_{n}\right) T x_{n}+\alpha_{n} T y_{n}, \\
y_{n}=\left(1-\beta_{n}\right) x_{n}+\beta_{n} T x_{n}, \quad n \in \mathbb{N},
\end{gathered}
$$

is known as the $S$ iteration process $[6,19]$.

Continuing the above trend, we will introduce and employ the following iterative schemes which are called Kirkmultistep-SP and Kirk-S iterations, respectively:

$$
\begin{gathered}
x_{0} \in X, \\
x_{n+1}=\sum_{i_{1}=0}^{s_{1}} \alpha_{n, i_{1}} T^{i_{1}} y_{n}^{1}, \\
y_{n}^{p}=\sum_{i_{p+1}=0}^{s_{p+1}} \beta_{n, i_{p+1}}^{p} T^{i_{p+1}} y_{n}^{p+1}, \quad p=\overline{1, k-2}, \\
y_{n}^{k-1}=\sum_{i_{k}=0}^{s_{k}} \beta_{n, i_{k}}^{k-1} T^{i_{k}} x_{n}, \quad k \geq 2, \quad \forall n \in \mathbb{N}, \\
x_{n+1}=\alpha_{n, 0} T x_{n}+\sum_{i_{1}=1}^{s_{1}} \alpha_{n, i_{1}} T^{i_{1}} y_{n}, \\
y_{n}=\sum_{i_{2}=0}^{s_{2}} \beta_{n, i_{2}} T^{i_{2}} x_{n}, \quad \forall n \in \mathbb{N},
\end{gathered}
$$

where $\sum_{i_{1}=0}^{s_{1}} \alpha_{n, i_{1}}=1, \sum_{i_{p+1}=0}^{s_{p+1}} \beta_{n, i_{p+1}}^{p}=1$ for $p=\overline{1, k-1}$; $\alpha_{n, i_{1}}, \beta_{n, i_{p+1}}^{p}$ are sequences in $[0,1]$ satisfying $\alpha_{n, i_{1}} \geq 0, \alpha_{n, 0} \neq 0$, $\beta_{n, i_{p+1}}^{p} \geq 0$, and $\beta_{n, 0}^{p} \neq 0$ for $p=\overline{1, k-1}$; and $s_{1}, s_{p+1}$ for $p=\overline{1, k-1}$ are fixed integers with $s_{1} \geq s_{2} \geq \cdots \geq s_{k}$.

By taking $k=3, k=2$, and $k=2$ with $s_{2}=0$ in (5) we obtain the Kirk-SP [30], a Kirk-two-step-Mann, and the KirkMann [31] iterative schemes, respectively. Also, (5) gives the usual Kirk iterative process [32] for $k=2$, with $s_{2}=0$ and $\alpha_{n, i_{1}}=\alpha_{i_{1}}$. If we put $s_{1}=1$ and $s_{p+1}=1, p=\overline{1, k-1}$ in (5) and (6), then we have the usual multistep-SP iteration (3) and $S$ iteration (4), respectively, with $\sum_{i_{1}=0}^{1} \alpha_{n, i_{1}}=1, \alpha_{n, 1}=\alpha_{n}$, $\sum_{i_{p+1}=0}^{1} \beta_{n, i_{p+1}}^{p}=1, \beta_{n, 1}^{p}=\beta_{n}^{p}, p=\overline{1, k-1}$. The SP iteration [25], the two-step Mann iteration [27], the Mann iteration
[23], the Krasnoselskij iteration [29], and the Picard iteration [18] schemes are special cases of the multistep-SP iterative scheme (3), as explained above. So we conclude that these are also special cases of the Kirk-multistep-SP iterative scheme (5).

We end this section with some definitions and lemmas which will be useful in proving our main results.

Definition 1 (see [33]). Let $X$ be a normed space. A mapping $T: X \rightarrow X$ is called contractive-like mapping if there exists $\lambda \in[0,1)$ such that

$$
\|T x-T y\| \leq \varphi(\|x-T x\|)+\lambda\|x-y\|, \quad \forall x, y \in X,
$$

where $\varphi: \mathbb{R}^{+} \rightarrow \mathbb{R}^{+}$is a monotone increasing function with $\varphi(0)=0$.

Remark 2. By taking $\varphi(t)=L t$ in (7), one can get contractive definition due to Osilike and Udomene [34]. Also, by putting $\varphi(t)=2 \lambda t$ in (7), condition (7) reduces to the contractive definition in [35]. In [35] it was shown that the class of these operators is wider than class of Zamfirescu operators given in [17], where $\lambda:=\max \{a, b /(1-b), c /(1-c)\}, \lambda \in[0,1)$ and $a$, $b$, and $c$ are real numbers satisfying $0<a<1,0<b$, and $c<1 / 2$.

Remark 3 (see $[20,28]$ ). A map satisfying (7) need not have a fixed point. However, using (7), it is obvious that if $T$ has a fixed point, then it is unique.

Definition 4 (see $[36,37]$ ). Let $X$ be a normed space, $T$ : $X \rightarrow X$ a mapping, and $\left\{x_{n}\right\}_{n=0}^{\infty} \subset X$ an iterative sequence generated by the iterative process (1) with limit point $q \in$ $F_{T}:=\{q \in X: q=T q\}$. Let $\left\{y_{n}\right\}_{n=0}^{\infty}$ be an arbitrary sequence in $X$ and set

$$
\varepsilon_{n}=\left\|y_{n+1}-f\left(T, y_{n}\right)\right\| \quad \text { for } n=0,1,2, \ldots .
$$

We will say that the iterative sequence $\left\{x_{n}\right\}_{n=0}^{\infty}$ is $T$-stable or stable with respect to $T$ if and only if

$$
\lim _{n \rightarrow \infty} \varepsilon_{n}=0 \Longleftrightarrow \lim _{n \rightarrow \infty} y_{n}=q .
$$

Lemma 5 (see [8]). If $\sigma$ is a real number such that $\sigma \in[0,1)$ and $\left\{\varepsilon_{n}\right\}_{n=0}^{\infty}$ is a sequence of nonnegative numbers such that $\lim _{n \rightarrow \infty} \varepsilon_{n}=0$, then, for any sequence of positive numbers $\left\{u_{n}\right\}_{n=0}^{\infty}$ satisfying

$$
u_{n+1} \leq \sigma u_{n}+\varepsilon_{n}, \quad \forall n \in \mathbb{N},
$$

one has $\lim _{n \rightarrow \infty} u_{n}=0$.

Lemma 6 (see [31]). Let $(X,\|\cdot\|)$ be a normed linear space and let $T$ be a self-map of $X$ satisfying (7). Let $\varphi: \mathbb{R}^{+} \rightarrow \mathbb{R}^{+}$be a subadditive, monotone increasing function such that $\varphi(0)=0$, $\varphi(L u) \leq L \varphi(u), L \geq 0, u \in \mathbb{R}^{+}$. Then, for all $i \in \mathbb{N}, L \geq 0$ and for all $x, y \in X$

$$
\left\|T^{i} x-T^{i} y\right\| \leq \sum_{j=1}^{i}\left(\begin{array}{c}
i \\
j
\end{array}\right) a^{i-j} \varphi^{j}(\|x-T x\|)+a^{i}\|x-y\|,
$$

where $a \in[0,1)$. 


\section{Main Results}

For simplicity we assume in the following four theorems that $X$ is a normed linear space, $T$ is a self map of $X$ satisfying the contractive condition (7) with some fixed point $q \in F_{T}$, and $\varphi: \mathbb{R}^{+} \rightarrow \mathbb{R}^{+}$is a subadditive monotone increasing function such that $\varphi(0)=0$ and $\varphi(L u) \leq L \varphi(u), L \geq 0, u \in \mathbb{R}^{+}$.

Theorem 7. Let $\left\{x_{n}\right\}_{n \in \mathbb{N}}$ be a sequence generated by the Kirkmultistep-SP iterative scheme (5). Then the iterative sequence $\left\{x_{n}\right\}_{n \in \mathbb{N}}$ converges strongly to $q$.

Proof. The uniqueness of $q$ follows from (7). We will now prove that $x_{n} \rightarrow q$.

Using Kirk-multistep-SP iterative process (5), condition (7), and Lemma 6, we get

$$
\begin{aligned}
& \left\|x_{n+1}-q\right\| \\
& =\left\|\sum_{i_{1}=0}^{s_{1}} \alpha_{n, i_{1}} T^{i_{1}} y_{n}^{1}-q\right\| \\
& =\left\|\alpha_{n, 0}\left(y_{n}^{1}-q\right)+\sum_{i_{1}=1}^{s_{1}} \alpha_{n, i_{1}}\left(T^{i_{1}} y_{n}^{1}-T^{i_{1}} q\right)\right\| \\
& \leq \alpha_{n, 0}\left\|y_{n}^{1}-q\right\|+\sum_{i_{1}=1}^{s_{1}} \alpha_{n, i_{1}}\left\|T^{i_{1}} y_{n}^{1}-T^{i_{1}} q\right\| \\
& \leq \alpha_{n, 0}\left\|y_{n}^{1}-q\right\| \\
& \quad+\sum_{i_{1}=1}^{s_{1}} \alpha_{n, i_{1}} \\
& \quad \times\left\{\sum_{j=1}^{i_{1}}\left(\begin{array}{c}
i_{1} \\
j
\end{array}\right) a^{i_{1}-j} \varphi^{j}(\|q-T q\|)+a^{i_{1}}\left\|y_{n}^{1}-q\right\|\right\} \\
& =\left(\sum_{i_{1}=0}^{s_{1}} \alpha_{n, i_{1}} a^{i_{1}}\right)\left\|y_{n}^{1}-q\right\|,
\end{aligned}
$$$$
\left\|y_{n}^{1}-q\right\|
$$$$
=\left\|\sum_{i_{2}=0}^{s_{2}} \beta_{n, i_{2}}^{1} T^{i_{2}} y_{n}^{2}-q\right\|
$$$$
=\left\|\sum_{i_{2}=0}^{s_{2}} \beta_{n, i_{2}}^{1}\left(T^{i_{2}} y_{n}^{2}-T^{i_{2}} q\right)\right\|
$$$$
\leq \beta_{n, 0}^{1}\left\|y_{n}^{2}-q\right\|+\sum_{i_{2}=1}^{s_{2}} \beta_{n, i_{2}}^{1}\left\|T^{i_{2}} y_{n}^{2}-T^{i_{2}} q\right\|
$$$$
\leq \beta_{n, 0}^{1}\left\|y_{n}^{2}-q\right\|
$$$$
+\sum_{i_{2}=1}^{s_{2}} \beta_{n, i_{2}}^{1}
$$

$$
\begin{gathered}
\times\left\{\sum_{j=1}^{i_{2}}\left(\begin{array}{c}
i_{2} \\
j
\end{array}\right) a^{i_{2}-j} \varphi^{j}(\|q-T q\|)+a^{i_{2}}\left\|y_{n}^{2}-q\right\|\right\} \\
=\left(\sum_{i_{2}=0}^{s_{2}} \beta_{n, i_{2}}^{1} a^{i_{2}}\right)\left\|y_{n}^{2}-q\right\| \\
\left\|y_{n}^{2}-q\right\| \leq\left(\sum_{i_{3}=0}^{s_{3}} \beta_{n, i_{3}}^{2} a^{i_{3}}\right)\left\|y_{n}^{3}-q\right\|
\end{gathered}
$$

By combining (12), (13), and (14) we obtain

$$
\begin{aligned}
\| x_{n+1} & -q \| \\
& \leq\left(\sum_{i_{1}=0}^{s_{1}} \alpha_{n, i_{1}} a^{i_{1}}\right)\left(\sum_{i_{2}=0}^{s_{2}} \beta_{n, i_{2}}^{1} a^{i_{2}}\right)\left(\sum_{i_{3}=0}^{s_{3}} \beta_{n, i_{3}}^{2} a^{i_{3}}\right)\left\|y_{n}^{3}-q\right\| .
\end{aligned}
$$

Continuing the above process we have

$$
\begin{aligned}
\left\|x_{n+1}-q\right\| \leq & \left(\sum_{i_{1}=0}^{s_{1}} \alpha_{n, i_{1}} a^{i_{1}}\right)\left(\sum_{i_{2}=0}^{s_{2}} \beta_{n, i_{2}}^{1} a^{i_{2}}\right) \cdots \\
& \left(\sum_{i_{k-1}=0}^{s_{k-1}} \beta_{n, i_{k-1}}^{k-2} a^{i_{k-1}}\right)\left\|y_{n}^{k-1}-q\right\| .
\end{aligned}
$$

Using again Kirk-multistep-SP iterative process (5), condition (7), and Lemma 6, we have

$$
\left\|y_{n}^{k-1}-q\right\| \leq\left(\sum_{i_{k}=0}^{s_{k}} \beta_{n, i_{k}}^{k-1} a^{i_{k}}\right)\left\|x_{n}-q\right\| .
$$

Substituting (17) into (16) we derive

$$
\begin{aligned}
\left\|x_{n+1}-q\right\| \leq & \left(\sum_{i_{1}=0}^{s_{1}} \alpha_{n, i_{1}} a^{i_{1}}\right)\left(\sum_{i_{2}=0}^{s_{2}} \beta_{n, i_{2}}^{1} a^{i_{2}}\right) \cdots \\
& \left(\sum_{i_{k}=0}^{s_{k}} \beta_{n, i_{k}}^{k-1} a^{i_{k}}\right)\left\|x_{n}-q\right\| .
\end{aligned}
$$

Since $a^{i_{k}} \in[0,1)$ and $\sum_{i_{1}=0}^{s_{1}} \alpha_{n, i_{1}}=1, \sum_{i_{p+1}=0}^{s_{p+1}} \beta_{n, i_{p+1}}^{p}=1$ for $p=\overline{1, k-1}$, then

$$
\begin{gathered}
\left(\sum_{i_{1}=0}^{s_{1}} \alpha_{n, i_{1}} a^{i_{1}}\right)\left(\sum_{i_{2}=0}^{s_{2}} \beta_{n, i_{2}}^{1} a^{i_{2}}\right) \cdots\left(\sum_{i_{k}=0}^{s_{k}} \beta_{n, i_{k}}^{k-1} a^{i_{k}}\right) \\
<\left(\sum_{i_{1}=0}^{s_{1}} \alpha_{n, i_{1}}\right)\left(\sum_{i_{2}=0}^{s_{2}} \beta_{n, i_{2}}^{1}\right) \cdots\left(\sum_{i_{k}=0}^{s_{k}} \beta_{n, i_{k}}^{k-1}\right)=1 .
\end{gathered}
$$

Hence, by an application of Lemma 5 to the inequality (18), we get $\lim _{n \rightarrow \infty} x_{n}=q$.

Theorem 8. Let $\left\{x_{n}\right\}_{n \in \mathbb{N}}$ be a sequence generated by the Kirkmultistep-SP iterative scheme (5). Then, the iterative sequence $\left\{x_{n}\right\}_{n \in \mathbb{N}}$ is T-stable. 
Proof. Let $\left\{y_{n}\right\}_{n \in \mathbb{N}} \subset X,\left\{u_{n}^{p}\right\}_{n \in \mathbb{N}}$, for $p=\overline{1, k-1}$, be arbitrary sequences in $X$. Let $\varepsilon_{n}=\left\|y_{n+1}-\sum_{i_{1}=0}^{s_{1}} \alpha_{n, i_{1}} T^{i_{1}} u_{n}^{1}\right\|, n=$ $0,1,2, \ldots$, where $u_{n}^{p}=\sum_{i_{p+1}=0}^{s_{p+1}} \beta_{n, i_{p+1}}^{p} T^{i_{p+1}} u_{n}^{p+1}, p=\overline{1, k-2}$, $u_{n}^{k-1}=\sum_{i_{k}=0}^{s_{k}} \beta_{n, i_{k}}^{k-1} T^{i_{k}} y_{n}, k \geq 2$, and let $\lim _{n \rightarrow \infty} \varepsilon_{n}=0$. Now we will prove that $\lim _{n \rightarrow \infty} y_{n}=q$.

It follows from (5) and Lemma 6 that

$$
\begin{aligned}
& \left\|y_{n+1}-q\right\| \\
& =\left\|y_{n+1}-\sum_{i_{1}=0}^{s_{1}} \alpha_{n, i_{1}} T^{i_{1}} u_{n}^{1}+\sum_{i_{1}=0}^{s_{1}} \alpha_{n, i_{1}} T^{i_{1}} u_{n}^{1}-q\right\| \\
& \leq\left\|y_{n+1}-\sum_{i_{1}=0}^{s_{1}} \alpha_{n, i_{1}} T^{i_{1}} u_{n}^{1}\right\|+\left\|\sum_{i_{1}=0}^{s_{1}} \alpha_{n, i_{1}} T^{i_{1}} u_{n}^{1}-q\right\| \\
& =\varepsilon_{n}+\left\|\sum_{i_{1}=0}^{s_{1}} \alpha_{n, i_{1}}\left(T^{i_{1}} u_{n}^{1}-T^{i_{1}} q\right)\right\| \\
& =\varepsilon_{n}+\left\|\alpha_{n, 0}\left(u_{n}^{1}-q\right)+\sum_{i_{1}=1}^{s_{1}} \alpha_{n, i_{1}}\left(T^{i_{1}} u_{n}^{1}-T^{i_{1}} q\right)\right\| \\
& \leq \varepsilon_{n}+\alpha_{n, 0}\left\|u_{n}^{1}-q\right\|+\sum_{i_{1}=1}^{s_{1}} \alpha_{n, i_{1}}\left\|T^{i_{1}} u_{n}^{1}-T^{i_{1}} q\right\| \\
& \leq \varepsilon_{n}+\alpha_{n, 0}\left\|u_{n}^{1}-q\right\| \\
& +\sum_{i_{1}=1}^{s_{1}} \alpha_{n, i_{1}} \\
& \times\left\{\sum_{j=1}^{i_{1}}\left(\begin{array}{c}
i_{1} \\
j
\end{array}\right) a^{i_{1}-j} \varphi^{j}(\|q-T q\|)+a^{i_{1}}\left\|u_{n}^{1}-q\right\|\right\} \\
& =\varepsilon_{n}+\left(\sum_{i_{1}=0}^{s_{1}} \alpha_{n, i_{1}} a^{i_{1}}\right)\left\|u_{n}^{1}-q\right\|,
\end{aligned}
$$

$\left\|u_{n}^{1}-q\right\|$

$$
\begin{aligned}
= & \left\|\sum_{i_{2}=0}^{s_{2}} \beta_{n, i_{2}}^{1} T^{i_{2}} u_{n}^{2}-q\right\| \\
= & \left\|\sum_{i_{2}=0}^{s_{2}} \beta_{n, i_{2}}^{1}\left(T^{i_{2}} u_{n}^{2}-T^{i_{2}} q\right)\right\| \\
\leq & \beta_{n, 0}^{1}\left\|u_{n}^{2}-q\right\|+\sum_{i_{2}=1}^{s_{2}} \beta_{n, i_{2}}^{1}\left\|T^{i_{2}} u_{n}^{2}-T^{i_{2}} q\right\| \\
\leq & \beta_{n, 0}^{1}\left\|u_{n}^{2}-q\right\| \\
& +\sum_{i_{2}=1}^{s_{2}} \beta_{n, i_{2}}^{1}
\end{aligned}
$$

$$
\begin{gathered}
\times\left\{\sum_{j=1}^{i_{2}}\left(\begin{array}{c}
i_{2} \\
j
\end{array}\right) a^{i_{2}-j} \varphi^{j}(\|q-T q\|)+a^{i_{2}}\left\|u_{n}^{2}-q\right\|\right\} \\
=\left(\sum_{i_{2}=0}^{s_{2}} \beta_{n, i_{2}}^{1} a^{i_{2}}\right)\left\|u_{n}^{2}-q\right\| \\
\left\|u_{n}^{2}-q\right\| \leq\left(\sum_{i_{3}=0}^{s_{3}} \beta_{n, i_{3}}^{2} a^{i_{3}}\right)\left\|u_{n}^{3}-q\right\|
\end{gathered}
$$

Combining (20), (21), and (22) we get

$$
\begin{aligned}
\left\|y_{n+1}-q\right\| \leq & \varepsilon_{n}+\left(\sum_{i_{1}=0}^{s_{1}} \alpha_{n, i_{1}} a^{i_{1}}\right)\left(\sum_{i_{2}=0}^{s_{2}} \beta_{n, i_{2}}^{1} a^{i_{2}}\right) \\
& \times\left(\sum_{i_{3}=0}^{s_{3}} \beta_{n, i_{3}}^{2} a^{i_{3}}\right)\left\|u_{n}^{3}-q\right\| .
\end{aligned}
$$

By induction

$$
\begin{gathered}
\left\|y_{n+1}-q\right\| \leq \varepsilon_{n}+\left(\sum_{i_{1}=0}^{s_{1}} \alpha_{n, i_{1}} a^{i_{1}}\right)\left(\sum_{i_{2}=0}^{s_{2}} \beta_{n, i_{2}}^{1} a^{i_{2}}\right) \cdots \\
\left(\sum_{i_{k-1}=0}^{s_{k-1}} \beta_{n, i_{k-1}}^{k-2} a^{i_{k-1}}\right)\left\|u_{n}^{k-1}-q\right\| .
\end{gathered}
$$

Again using (5) and Lemma 6 we have

$$
\left\|u_{n}^{k-1}-q\right\| \leq\left(\sum_{i_{k}=0}^{s_{k}} \beta_{n, i_{k}}^{k-1} a^{i_{k}}\right)\left\|y_{n}-q\right\| .
$$

Substituting (25) into (24) we derive

$$
\begin{gathered}
\left\|y_{n+1}-q\right\| \leq \varepsilon_{n}+\left(\sum_{i_{1}=0}^{s_{1}} \alpha_{n, i_{1}} a^{i_{1}}\right)\left(\sum_{i_{2}=0}^{s_{2}} \beta_{n, i_{2}}^{1} a^{i_{2}}\right) \cdots \\
\left(\sum_{i_{k}=0}^{s_{k}} \beta_{n, i_{k}}^{k-1} a^{i_{k}}\right)\left\|y_{n}-q\right\| .
\end{gathered}
$$

Define

$$
\sigma:=\left(\sum_{i_{1}=0}^{s_{1}} \alpha_{n, i_{1}} a^{i_{1}}\right)\left(\sum_{i_{2}=0}^{s_{2}} \beta_{n, i_{2}}^{1} a^{i_{2}}\right) \ldots\left(\sum_{i_{k}=0}^{s_{k}} \beta_{n, i_{k}}^{k-1} a^{i_{k}}\right) .
$$

We now show that $\sigma \in(0,1)$. Since $a^{i_{k}} \in[0,1), \alpha_{n, 0}>0$, $\sum_{i_{1}=0}^{s_{1}} \alpha_{n, i_{1}}=1$, and $\sum_{i_{p+1}=0}^{s_{p+1}} \beta_{n, i_{p+1}}^{p}=1$ for $p=\overline{1, k-1}$, we have

$$
\sigma<\left(\sum_{i_{1}=0}^{s_{1}} \alpha_{n, i_{1}}\right)\left(\sum_{i_{2}=0}^{s_{2}} \beta_{n, i_{2}}^{1}\right) \cdots\left(\sum_{i_{k}=0}^{s_{k}} \beta_{n, i_{k}}^{k-1}\right)=1 .
$$

Therefore, an application of Lemma 5 to (26) yields $\lim _{n \rightarrow \infty} y_{n}=q$.

Now suppose that $\lim _{n \rightarrow \infty} y_{n}=q$. Then, we will show that $\lim _{n \rightarrow \infty} \varepsilon_{n}=0$. 
Journal of Function Spaces

5

Using Lemma 6 we have

$\varepsilon_{n}=\left\|y_{n+1}-\sum_{i_{1}=0}^{s_{1}} \alpha_{n, i_{1}} T^{i_{1}} u_{n}^{1}\right\|$

$$
\begin{aligned}
& \leq\left\|y_{n+1}-q\right\|+\left\|q-\sum_{i_{1}=0}^{s_{1}} \alpha_{n, i_{1}} T^{i_{1}} u_{n}^{1}\right\| \\
& =\left\|y_{n+1}-q\right\|
\end{aligned}
$$

$$
\begin{aligned}
& +\left\|\alpha_{n, 0}\left(q-u_{n}^{1}\right)+\sum_{i_{1}=1}^{s_{1}} \alpha_{n, i_{1}}\left(T^{i_{1}} q-T^{i_{1}} u_{n}^{1}\right)\right\| \\
& \leq\left\|y_{n+1}-q\right\|+\alpha_{n, 0}\left\|u_{n}^{1}-q\right\|
\end{aligned}
$$

$+\sum_{i_{1}=1}^{s_{1}} \alpha_{n, i_{1}}\left\|T^{i_{1}} q-T^{i_{1}} u_{n}^{1}\right\|$

$$
\leq\left\|y_{n+1}-q\right\|+\alpha_{n, 0}\left\|q-u_{n}^{1}\right\|
$$

$$
+\sum_{i_{1}=1}^{s_{1}} \alpha_{n, i_{1}}
$$

$$
\times\left\{\sum_{j=1}^{i_{1}}\left(\begin{array}{c}
i_{1} \\
j
\end{array}\right) a^{i_{1}-j} \varphi^{j}(\|q-T q\|)+a^{i_{1}}\left\|q-u_{n}^{1}\right\|\right\}
$$

$\leq\left\|y_{n+1}-q\right\|+\left(\sum_{i_{1}=0}^{s_{1}} \alpha_{n, i_{1}} a^{i_{1}}\right)\left\|q-u_{n}^{1}\right\|$,

$$
\begin{aligned}
\| q- & u_{n}^{1} \| \\
= & \left\|q-\sum_{i_{2}=0}^{s_{2}} \beta_{n, i_{2}}^{1} T^{i_{2}} u_{n}^{2}\right\| \\
= & \left\|\beta_{n, 0}^{1}\left(q-u_{n}^{2}\right)+\sum_{i_{2}=1}^{s_{2}} \beta_{n, i_{2}}^{1}\left(T^{i_{2}} q-T^{i_{2}} u_{n}^{2}\right)\right\| \\
\leq & \beta_{n, 0}^{1}\left\|q-u_{n}^{2}\right\|+\sum_{i_{2}=1}^{s_{2}} \beta_{n, i_{2}}^{1}\left\|T^{i_{2}} q-T^{i_{2}} u_{n}^{2}\right\| \\
\leq & \beta_{n, 0}^{1}\left\|q-u_{n}^{2}\right\| \\
& \left.+\sum_{i_{2}=1}^{s_{2}} \beta_{n, i_{2}}^{1}{ }^{i_{2}}{ }^{i_{2}}\left(\begin{array}{c}
i_{2} \\
j
\end{array}\right) a^{i_{2}-j} \varphi^{j}(\|q-T q\|)+a^{i_{2}}\left\|q-u_{n}^{2}\right\|\right\} \\
\leq & \left(\sum_{i_{2}=0}^{s_{2}} \beta_{n, i_{2}}^{1} a^{i_{2}}\right)\left\|q-u_{n}^{2}\right\|, \\
& \left\|q-u_{n}^{2}\right\| \leq\left(\sum_{i_{3}=0}^{s_{3}} \beta_{n, i_{3}}^{2} a^{i_{3}}\right)\left\|q-u_{n}^{3}\right\| .
\end{aligned}
$$

Combining (29), (30), and (31) we obtain

$$
\begin{aligned}
\varepsilon_{n} \leq & \left\|y_{n+1}-q\right\|+\left(\sum_{i_{1}=0}^{s_{1}} \alpha_{n, i_{1}} a^{i_{1}}\right)\left(\sum_{i_{2}=0}^{s_{2}} \beta_{n, i_{2}}^{1} a^{i_{2}}\right) \\
& \times\left(\sum_{i_{3}=0}^{s_{3}} \beta_{n, i_{3}}^{2} a^{i_{3}}\right)\left\|q-u_{n}^{3}\right\| .
\end{aligned}
$$

Thus, by induction, we get

$$
\begin{gathered}
\varepsilon_{n} \leq\left\|y_{n+1}-q\right\|+\left(\sum_{i_{1}=0}^{s_{1}} \alpha_{n, i_{1}} a^{i_{1}}\right)\left(\sum_{i_{2}=0}^{s_{2}} \beta_{n, i_{2}}^{1} a^{i_{2}}\right) \cdots \\
\left(\sum_{i_{k-1}=0}^{s_{k-1}} \beta_{n, i_{k-1}}^{k-2} a^{i_{k-1}}\right)\left\|q-u_{n}^{k-1}\right\| .
\end{gathered}
$$

Again using (5) and Lemma 6 we have

$$
\left\|q-u_{n}^{k-1}\right\| \leq\left(\sum_{i_{k}=0}^{s_{k}} \beta_{n, i_{k}}^{k-1} a^{i_{k}}\right)\left\|y_{n}-q\right\| .
$$

Substituting (34) into (33) we derive

$$
\begin{gathered}
\varepsilon_{n} \leq\left\|y_{n+1}-q\right\|+\left(\sum_{i_{1}=0}^{s_{1}} \alpha_{n, i_{1}} a^{i_{1}}\right)\left(\sum_{i_{2}=0}^{s_{2}} \beta_{n, i_{2}}^{1} a^{i_{2}}\right) \cdots \\
\left(\sum_{i_{k}=0}^{s_{k}} \beta_{n, i_{k}}^{k-1} a^{i_{k}}\right)\left\|y_{n}-q\right\| .
\end{gathered}
$$

Again define

$$
\sigma:=\left(\sum_{i_{1}=0}^{s_{1}} \alpha_{n, i_{1}} a^{i_{1}}\right)\left(\sum_{i_{2}=0}^{s_{2}} \beta_{n, i_{2}}^{1} a^{i_{2}}\right) \cdots\left(\sum_{i_{k}=0}^{s_{k}} \beta_{n, i_{k}}^{k-1} a^{i_{k}}\right) .
$$

Using the same argument as that of the first part of the proof we obtain $\sigma \in(0,1)$.

Hence (35) becomes

$$
\varepsilon_{n} \leq\left\|y_{n+1}-q\right\|+\sigma\left\|y_{n}-q\right\|
$$

It therefore follows from assumption $\lim _{n \rightarrow \infty} y_{n}=q$ that $\varepsilon_{n} \rightarrow 0$ as $n \rightarrow \infty$.

Theorem 9. Let $\left\{x_{n}\right\}_{n \in \mathbb{N}}$ be a sequence generated by the Kirk$S$ iterative scheme (6). Then, the iterative sequence $\left\{x_{n}\right\}_{n \in \mathbb{N}}$ converges strongly to $q$.

Proof. The uniqueness of $q$ follows from (7). We will now prove that $x_{n} \rightarrow q$. 
Using Kirk-S iterative process (6), condition (7), and Lemma 6, we get

$$
\begin{aligned}
\left\|x_{n+1}-q\right\| & \left\|\alpha_{n, 0}\left(T x_{n}-T q\right)+\sum_{i_{1}=1}^{s_{1}} \alpha_{n, i_{1}}\left(T^{i_{1}} y_{n}-T^{i_{1}} q\right)\right\| \\
\leq & \alpha_{n, 0}\left\|T x_{n}-T q\right\|+\sum_{i_{1}=1}^{s_{1}} \alpha_{n, i_{1}}\left\|T^{i_{1}} y_{n}-T^{i_{1}} q\right\| \\
\leq & a \alpha_{n, 0}\left\|x_{n}-q\right\| \\
& +\sum_{i_{1}=1}^{s_{1}} \alpha_{n, i_{1}}\left\{\sum_{j=1}^{i_{1}}\left(\begin{array}{c}
i_{1} \\
j
\end{array}\right) a^{i_{1}-j} \varphi^{j}(\|q-T q\|)+a^{i_{1}}\left\|y_{n}-q\right\|\right\} \\
= & a \alpha_{n, 0}\left\|x_{n}-q\right\|+\left(\sum_{i_{1}=1}^{s_{1}} \alpha_{n, i_{1}} a^{i_{1}}\right)\left\|y_{n}-q\right\|,
\end{aligned}
$$

$$
\begin{aligned}
\left\|y_{n}-q\right\| & \left\|\sum_{i_{2}=0}^{s_{2}} \beta_{n, i_{2}}\left(T^{i_{2}} x_{n}-T^{i_{2}} q\right)\right\| \\
\leq & \beta_{n, 0}\left\|x_{n}-q\right\|+\sum_{i_{2}=1}^{s_{2}} \beta_{n, i_{2}}\left\|T^{i_{2}} x_{n}-T^{i_{2}} q\right\| \\
\leq & \beta_{n, 0}\left\|x_{n}-q\right\| \\
& +\sum_{i_{2}=1}^{s_{2}} \beta_{n, i_{2}}\left\{\sum_{j=1}^{i_{2}}\left(\begin{array}{c}
i_{2} \\
j
\end{array}\right) a^{i_{2}-j} \varphi^{j}(\|q-T q\|)+a^{i_{2}}\left\|x_{n}-q\right\|\right\} \\
= & \left(\sum_{i_{2}=0}^{s_{2}} \beta_{n, i_{2}} a^{i_{2}}\right)\left\|x_{n}-q\right\| .
\end{aligned}
$$

Substituting (39) into (38) we obtain

$$
\begin{aligned}
\| x_{n+1} & -q \| \\
\leq & \left.\leq a \alpha_{n, 0}+\left(\sum_{i_{1}=1}^{s_{1}} \alpha_{n, i_{1}} a^{i_{1}}\right)\left(\sum_{i_{2}=0}^{s_{2}} \beta_{n, i_{2}} a^{i_{2}}\right)\right]\left\|x_{n}-q\right\| .
\end{aligned}
$$

Since $a^{i_{k}} \in[0,1)$ and $\sum_{i_{1}=0}^{s_{1}} \alpha_{n, i_{1}}=\sum_{i_{2}=0}^{s_{2}} \beta_{n, i_{2}}=1$ with $\alpha_{n, 0} \neq 0$, $\beta_{n, 0} \neq 0$,

$$
\begin{aligned}
a \alpha_{n, 0} & +\left(\sum_{i_{1}=1}^{s_{1}} \alpha_{n, i_{1}} a^{i_{1}}\right)\left(\sum_{i_{2}=0}^{s_{2}} \beta_{n, i_{2}} a^{i_{2}}\right) \\
& <\alpha_{n, 0}+\left(\sum_{i_{1}=1}^{s_{1}} \alpha_{n, i_{1}}\right)\left(\sum_{i_{2}=0}^{s_{2}} \beta_{n, i_{2}}\right) \\
& =\sum_{i_{1}=0}^{s_{1}} \alpha_{n, i_{1}}=1 .
\end{aligned}
$$

Utilizing (41) and Lemma 5, (40) yields $\lim _{n \rightarrow \infty} x_{n}=q$.
Theorem 10. Let $\left\{x_{n}\right\}_{n \in \mathbb{N}}$ be a sequence generated by the Kirk$S$ iterative scheme (6). Then, the iterative sequence $\left\{x_{n}\right\}_{n \in \mathbb{N}}$ is T-stable.

Proof. Let $\left\{y_{n}\right\}_{n \in \mathbb{N}} \subset X, \varepsilon_{n}=\| y_{n+1}-\alpha_{n, 0} T y_{n}-$ $\sum_{i_{1}=1}^{s_{1}} \alpha_{n, i_{1}} T^{i_{1}} u_{n} \|, n=0,1,2, \ldots$, and $u_{n}=\sum_{i_{2}=0}^{s_{2}} \beta_{n, i_{2}} T^{i_{2}} y_{n}$. Assume that $\lim _{n \rightarrow \infty} \varepsilon_{n}=0$. Now we will prove that $\lim _{n \rightarrow \infty} y_{n}=q$.

It follows from (6) and Lemma 6 that

$$
\begin{aligned}
\left\|y_{n+1}-q\right\| & \left\|y_{n+1}-\alpha_{n, 0} T y_{n}-\sum_{i_{1}=1}^{s_{1}} \alpha_{n, i_{1}} T^{i_{1}} u_{n}\right\| \\
& +\left\|\alpha_{n, 0} T y_{n}+\sum_{i_{1}=1}^{s_{1}} \alpha_{n, i_{1}} T^{i_{1}} u_{n}-q\right\| \\
= & \varepsilon_{n}+\left\|\alpha_{n, 0}\left(T y_{n}-T q\right)+\sum_{i_{1}=1}^{s_{1}} \alpha_{n, i_{1}}\left(T^{i_{1}} u_{n}-T^{i_{1}} q\right)\right\| \\
\leq & \varepsilon_{n}+a \alpha_{n, 0}\left\|y_{n}-q\right\|+\sum_{i_{1}=1}^{s_{1}} \alpha_{n, i_{1}}\left\|T^{i_{1}} u_{n}-T^{i_{1}} q\right\| \\
\leq & \varepsilon_{n}+a \alpha_{n, 0}\left\|y_{n}-q\right\| \\
& +\sum_{i_{1}=1}^{s_{1}} \alpha_{n, i_{1}}\left\{\sum_{j=1}^{i_{1}}\left(\begin{array}{c}
i_{1} \\
j
\end{array}\right) a^{i_{1}-j} \varphi^{j}(\|q-T q\|)+a^{i_{1}}\left\|u_{n}-q\right\|\right\} \\
= & \varepsilon_{n}+a \alpha_{n, 0}\left\|y_{n}-q\right\|+\left(\sum_{i_{1}=1}^{s_{1}} \alpha_{n, i_{1}} a^{i_{1}}\right)\left\|u_{n}-q\right\|,
\end{aligned}
$$

$$
\left\|u_{n}-q\right\| \leq\left(\sum_{i_{2}=0}^{s_{2}} \beta_{n, i_{2}} a^{i_{2}}\right)\left\|y_{n}-q\right\| .
$$

Combining (42) and (43) we have

$$
\begin{aligned}
\left\|y_{n+1}-q\right\| \leq & \varepsilon_{n}+\left[a \alpha_{n, 0}+\left(\sum_{i_{1}=1}^{s_{1}} \alpha_{n, i_{1}} a^{i_{1}}\right)\left(\sum_{i_{2}=0}^{s_{2}} \beta_{n, i_{2}} a^{i_{2}}\right)\right] \\
& \times\left\|y_{n}-q\right\| .
\end{aligned}
$$

Define

$$
\sigma:=a \alpha_{n, 0}+\left(\sum_{i_{1}=1}^{s_{1}} \alpha_{n, i_{1}} a^{i_{1}}\right)\left(\sum_{i_{2}=0}^{s_{2}} \beta_{n, i_{2}} a^{i_{2}}\right)
$$


We now show that $\sigma \in(0,1)$. Since $a^{i_{k}} \in[0,1), \alpha_{n, 0}>0$, $\sum_{i_{1}=0}^{s_{1}} \alpha_{n, i_{1}}=1$, and $\sum_{i_{2}=0}^{s_{2}} \beta_{n, i_{2}} a^{i_{2}}$, we obtain

$$
\begin{aligned}
\sigma & <\alpha_{n, 0}+\left(\sum_{i_{1}=1}^{s_{1}} \alpha_{n, i_{1}}\right)\left(\sum_{i_{2}=0}^{s_{2}} \beta_{n, i_{2}}\right) \\
& =\sum_{i_{1}=0}^{s_{1}} \alpha_{n, i_{1}} \\
& =1 .
\end{aligned}
$$

Thus, (44) becomes

$$
\left\|y_{n+1}-q\right\| \leq \eta\left\|y_{n}-q\right\|+\varepsilon_{n} .
$$

Therefore, an application of Lemma 5 to (47) leads to $\lim _{n \rightarrow \infty} y_{n}=q$.

Now suppose that $\lim _{n \rightarrow \infty} y_{n}=q$. Then, we will show that $\lim _{n \rightarrow \infty} \varepsilon_{n}=0$.

Using Lemma 6 we have

$$
\begin{aligned}
& \varepsilon_{n}=\left\|y_{n+1}-\alpha_{n, 0} T y_{n}-\sum_{i_{1}=1}^{s_{1}} \alpha_{n, i_{1}} T^{i_{1}} u_{n}\right\| \\
& \leq\left\|y_{n+1}-q\right\| \\
&+\left\|q-\alpha_{n, 0} T y_{n}-\sum_{i_{1}=1}^{s_{1}} \alpha_{n, i_{1}} T^{i_{1}} u_{n}\right\| \\
&=\left\|y_{n+1}-q\right\| \\
&+\left\|\alpha_{n, 0}\left(T q-T y_{n}\right)+\sum_{i_{1}=1}^{s_{1}} \alpha_{n, i_{1}}\left(T^{i_{1}} q-T^{i_{1}} u_{n}\right)\right\| \\
& \leq\left\|y_{n+1}-q\right\|+a \alpha_{n, 0}\left\|y_{n}-q\right\| \\
&+\sum_{i_{1}=1}^{s_{1}} \alpha_{n, i_{1}}\left\|T^{i_{1}} q-T^{i_{1}} u_{n}\right\| \\
& \leq\left\|y_{n+1}-q\right\|+a \alpha_{n, 0}\left\|y_{n}-q\right\| \\
&+\sum_{i_{1}=1}^{s_{1}} \alpha_{n, i_{1}}\left\{\sum_{j=1}^{i_{1}}\left(\begin{array}{c}
i_{1} \\
j
\end{array}\right) a^{i_{1}-j} \varphi^{j}(\|q-T q\|)+a^{i_{1}}\left\|q-u_{n}\right\|\right\} \\
& \leq\left\|y_{n+1}-q\right\|+a \alpha_{n, 0}\left\|y_{n}-q\right\|+\left(\sum_{i_{1}=1}^{s_{1}} \alpha_{n, i_{1}} a^{i_{1}}\right)\left\|q-u_{n}\right\|, \\
& \quad\left\|q-u_{n}\right\| \leq\left(\sum_{i_{2}=0}^{s_{2}} \beta_{n, i_{2}} a^{i_{2}}\right)\left\|y_{n}-q\right\| .
\end{aligned}
$$

Substituting (49) into (48) we get

$$
\begin{aligned}
\varepsilon_{n} \leq & \left\|y_{n+1}-q\right\|+\left[a \alpha_{n, 0}+\left(\sum_{i_{1}=1}^{s_{1}} \alpha_{n, i_{1}} a^{i_{1}}\right)\left(\sum_{i_{2}=0}^{s_{2}} \beta_{n, i_{2}} a^{i_{2}}\right)\right] \\
& \times\left\|y_{n}-q\right\| .
\end{aligned}
$$

Again define

$$
\sigma:=a \alpha_{n, 0}+\left(\sum_{i_{1}=1}^{s_{1}} \alpha_{n, i_{1}} a^{i_{1}}\right)\left(\sum_{i_{2}=0}^{s_{2}} \beta_{n, i_{2}} a^{i_{2}}\right) .
$$

Using the same argument as that of the first part of the proof we obtain $\sigma \in(0,1)$.

Hence (50) becomes

$$
\varepsilon_{n} \leq\left\|y_{n+1}-q\right\|+\eta\left\|y_{n}-q\right\| .
$$

It therefore follows from assumption $\lim _{n \rightarrow \infty} y_{n}=q$ that $\varepsilon_{n} \rightarrow 0$ as $n \rightarrow \infty$.

Remark 11. Theorem 7 is a generalization and extension of Theorem 2.1 of [38], Theorem 2.1 of [39], Theorem 1 of [20], and Theorem 2.4 of [30]. Theorems 8 is a generalization and extension of Theorem 3.6 of [38] and Theorem 3 of [40]. Theorem 9 is a generalization and extension of Theorem 8 of [41] and Theorem 3 of [20].

\section{Conflict of Interests}

The authors declare that there is no conflict of interests regarding the publication of this paper.

\section{Acknowledgment}

This research has been supported by Yıldız Technical University Scientific Research Projects Coordination Department, Project no. BAPK 2012-07-03-DOP02.

\section{References}

[1] K. C. Border, Fixed Point Theorems with Applications to Economics and Game Theory, Cambridge University Press, Cambridge, UK, 1985.

[2] M. Droste, W. Kuich, and H. Vogler, Eds., Handbook of Weighted Automata. EATCS Mono-Graphs in Theoretical Computer Science, Springer, Berlin, Germany, 2009.

[3] E. A. Ok, Real Analysis with Economic Applications, Princeton University Press, Princeton, NJ, USA, 2007.

[4] N. Radde, "Fixed point characterization of biological networks with complex graph topology," Bioinformatics, vol. 26, no. 22, Article ID btq517, pp. 2874-2880, 2010.

[5] G. Q. Wang and S. S. Cheng, "Fixed point theorems arising from seeking steady states of neural networks," Applied Mathematical Modelling, vol. 33, no. 1, pp. 499-506, 2009.

[6] R. P. Agarwal, D. O'Regan, and D. R. Sahu, Fixed Point Theory for Lipschitzian Type-Mappings with Applications, Springer, New York, NY, USA, 2009. 
[7] S. Banach, "Sur les opérations dans les ensembles abstraits et leur application aux équations intégrales," Fundamenta Mathematicae, vol. 3, pp. 133-181, 1922.

[8] V. Berinde, Iterative Approximation of Fixed Points, Springer, Berlin, Germany, 2007.

[9] S. K. Chatterjea, "Fixed point theorems," Comptes Rendus de l'Academie Bulgare des Sciences, vol. 25, pp. 727-730, 1972.

[10] L. B. Ciric, "A generalization of Banach's contraction principle," Proceedings of the American Mathematical Society, vol. 45, pp. 267-273, 1974.

[11] M. Ertürk and V. Karakaya, "n-tuplet fixed point theorems for contractive type mappings in partially ordered metric spaces," Journal of Inequalities and Applications, vol. 2013, p. 196, 2013.

[12] R. Kannan, "Some results on fixed points," Bulletin of Calcutta Mathematical Society, vol. 60, pp. 71-76, 1968.

[13] M. A. Khamsi and W. A. Kirk, An Introduction to Metric Spaces and Fixed Point Theory, PAAM Monographs in Pure and Applied Mathematics, Wiley, New York, NY, USA, 2001.

[14] M. Mursaleen, S. A. Mohiuddine, and R. P. Agarwal, "Corrigendum to 'coupled fixed point theorems for $\alpha-\psi$-contractive type mappings in partially ordered metric spaces"' Fixed Point Theory and Applications, vol. 2013, p. 127, 2013.

[15] M. Mursaleen, S. A. Mohiuddine, and R. P. Agarwal, "Coupled fixed point theorems for $\alpha-\psi$-contractive type mappings in partially ordered metric spaces," Fixed Point Theory and Applications, vol. 2012, p. 228, 2012.

[16] R. Saadati, S. M. Vaezpour, P. Vetro, and B. E. Rhoades, "Fixed point theorems in generalized partially ordered Gmetric spaces," Mathematical and Computer Modelling, vol. 52, no. 5-6, pp. 797-801, 2010.

[17] T. Zamfirescu, "Fix point theorems in metric spaces," Archiv der Mathematik, vol. 23, no. 1, pp. 292-298, 1972.

[18] E. Picard, "Memoire sur la theorie des equations aux derivees partielles et la methode des approximations successives," Journal de Mathématiques Pures et Appliquées, vol. 6, pp. 145-210, 1890.

[19] R. P. Agarwal, D. O’Regan, and D. R. Sahu, "Iterative construction of fixed points of nearly asymp- totically nonexpansive mappings," Journal of Nonlinear and Convex Analysis, vol. 8, no. 1, pp. 61-79, 2007.

[20] F. Gürsoy, V. Karakaya, and B. E. Rhoades, "Data dependence results of new multistep and S-iterative schemes for contractivelike operators," Fixed Point Theory and Applications, vol. 2013, p. 76, 2013.

[21] S. Ishikawa, "Fixed points by a new iteration method," Proceedings of the American Mathematical Society, vol. 44, pp. 147-150, 1974.

[22] V. Karakaya, F. Gürsoy, K. Dogan, and M. Ertürk, "Data dependence results for multistep and CR iterative schemes in the class of contractive-like operators," Abstract and Applied Analysis, vol. 2013, Article ID 381980, 7 pages, 2013.

[23] W. R. Mann, "Mean value methods in iterations," Proceedings of the American Mathematical Society, vol. 4, pp. 506-510, 1953.

[24] M. A. Noor, "New approximation schemes for general variational inequalities," Journal of Mathematical Analysis and Applications, vol. 251, no. 1, pp. 217-229, 2000.

[25] W. Phuengrattana and S. Suantai, "On the rate of convergence of Mann, Ishikawa, Noor and SP-iterations for continuous functions on an arbitrary interval," Journal of Computational and Applied Mathematics, vol. 235, no. 9, pp. 3006-3014, 2011.
[26] B. E. Rhoades and S. M. Soltuz, "The equivalence between Mann-Ishikawa iterations and multistep iteration," Nonlinear Analysis, Theory, Methods and Applications, vol. 58, no. 1-2, pp. 219-228, 2004.

[27] S. Thianwan, "Common fixed points of new iterations for two asymptotically nonexpansive nonself-mappings in a Banach space," Journal of Computational and Applied Mathematics, vol. 224, no. 2, pp. 688-695, 2009.

[28] F. Gürsoy, V. Karakaya, and B. E. Rhoades, “The equivalence among new multistep iteration, S-iteration and some other iterative schemes," http://arxiv.org/abs/1211.5701. In press.

[29] M. A. Krasnoselkij, “Two remarks on the method of successive approximations," Uspehi Matematicheskih Nauk, vol. 63, no. 1, pp. 123-127, 1955.

[30] N. Hussain, R. Chugh, V. Kumar, and A. Rafiq, "On the rate of convergence of Kirk-type iterative schemes," Journal of Applied Mathematics, vol. 2012, Article ID 526503, 22 pages, 2012.

[31] M. O. Olatinwo, "Some stability results for two hybrid fixed point iterative algorithms in normed linear space," Matematicki Vesnik, vol. 61, no. 4, pp. 247-256, 2009.

[32] W. A. Kirk, "On successive approximations for nonexpansive mappings in Banach spaces," Glasgow Mathematical Journal, vol. 12, pp. 6-9, 1971.

[33] C. O. Imoru and M. O. Olatinwo, "On the stability of Picard and Mann iteration processes," Carpathian Journal of Mathematics, vol. 19, no. 2, pp. 155-160, 2003.

[34] M. O. Osilike and A. Udomene, "Short proofs of stability results for fixed point iteration procedures for a class of contractivetype mappings," Indian Journal of Pure and Applied Mathematics, vol. 30, no. 12, pp. 1229-1234, 1999.

[35] V. Berinde, "On the convergence of the Ishikawa iteration in the class of quasi contractive operators," Acta Mathematica Universitatis Comenianae, vol. 73, no. 1, pp. 119-126, 2004.

[36] A. M. Harder and T. L. Hicks, "A stable iteration procedure for nonexpansive mappings," Mathematica Japonica, vol. 33, pp. 687-692, 1988.

[37] A. M. Harder and T. L. Hicks, "Stability results for fixed point iteration procedures," Mathematica Japonica, vol. 33, pp. 693706, 1988.

[38] İ. Yıldırım, M. Özdemir, and H. Kızıltunç, “On the convergence of a new two-step iteration in the class of quasi-contractive operators," International Journal of Mathematical Analysis, vol. 3, pp. 1881-1892, 2009.

[39] R. Chugh and V. Kumar, "Strong convergence of SP iterative scheme for quasi-contractive operators in Banach spaces," International Journal of Applied Mathematics and Computer Science, vol. 31, pp. 21-27, 2011.

[40] F. Gürsoy, V. Karakaya, and B. E. Rhoades, "Some convergence and stability results for the Kirk multistep and Kirk-Sp fixed point iterative algorithms," Abstract and Applied Analysis, 2013.

[41] N. Hussain, A. Rafiq, B. Damjanovic, and R. Lazovic, "On rate of convergence of various iterative schemes," Fixed Point Theory and Applications, vol. 2011, p. 45, 2011. 


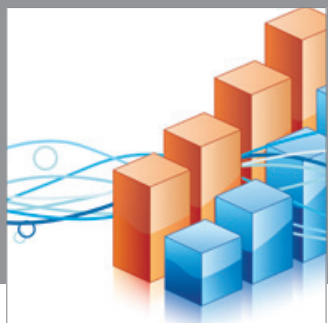

Advances in

Operations Research

mansans

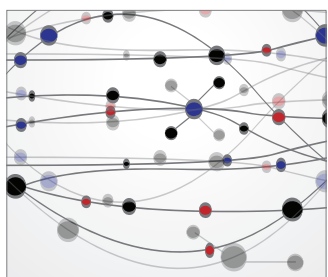

The Scientific World Journal
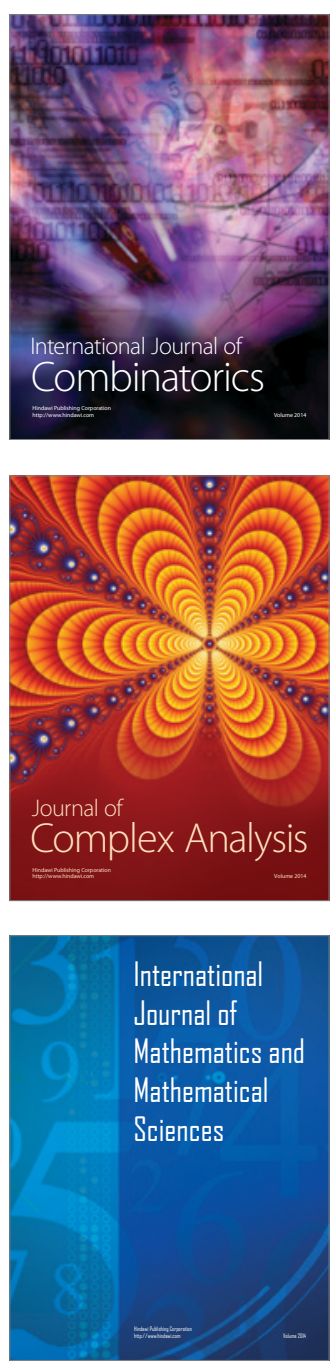
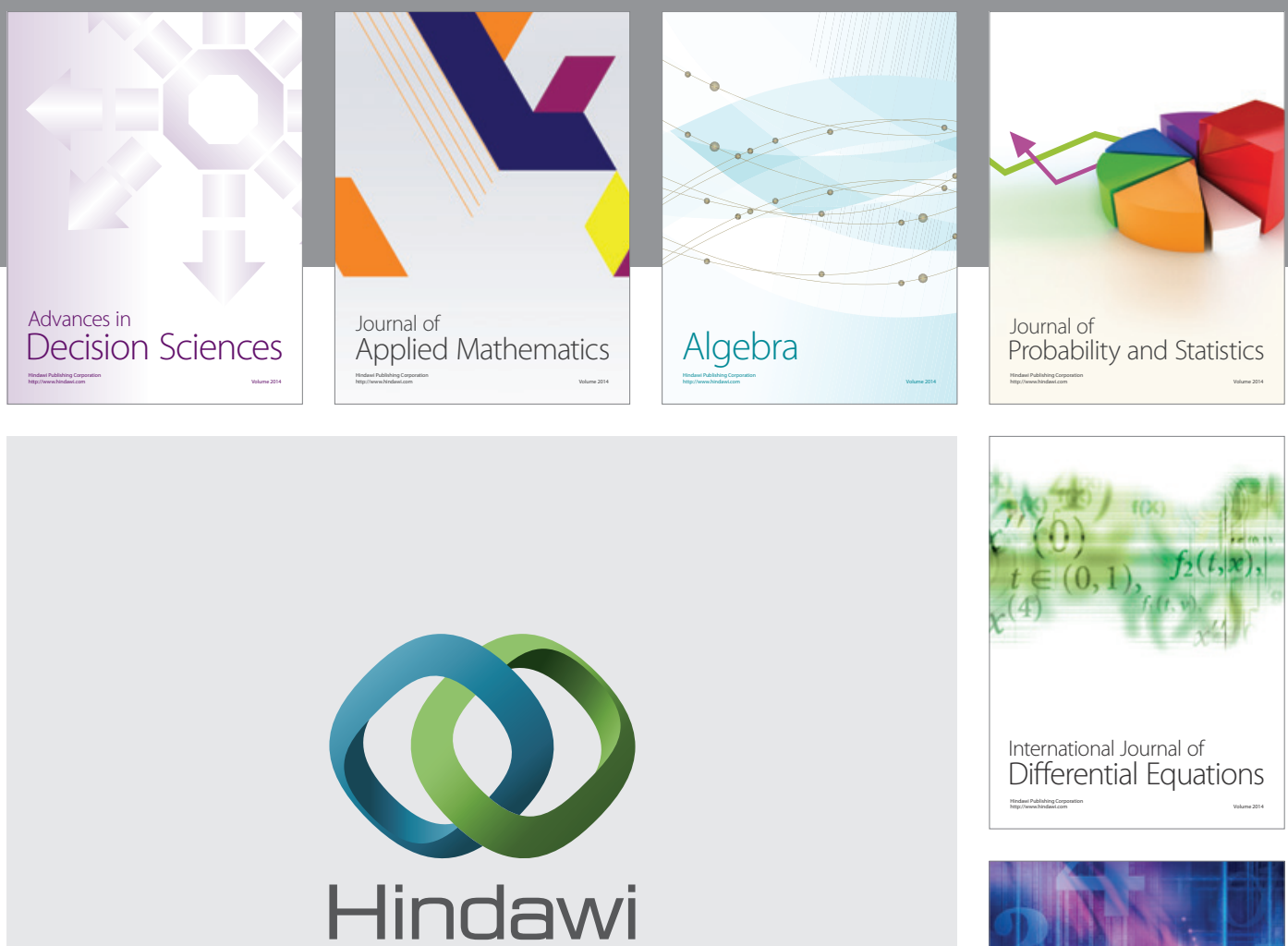

Submit your manuscripts at http://www.hindawi.com
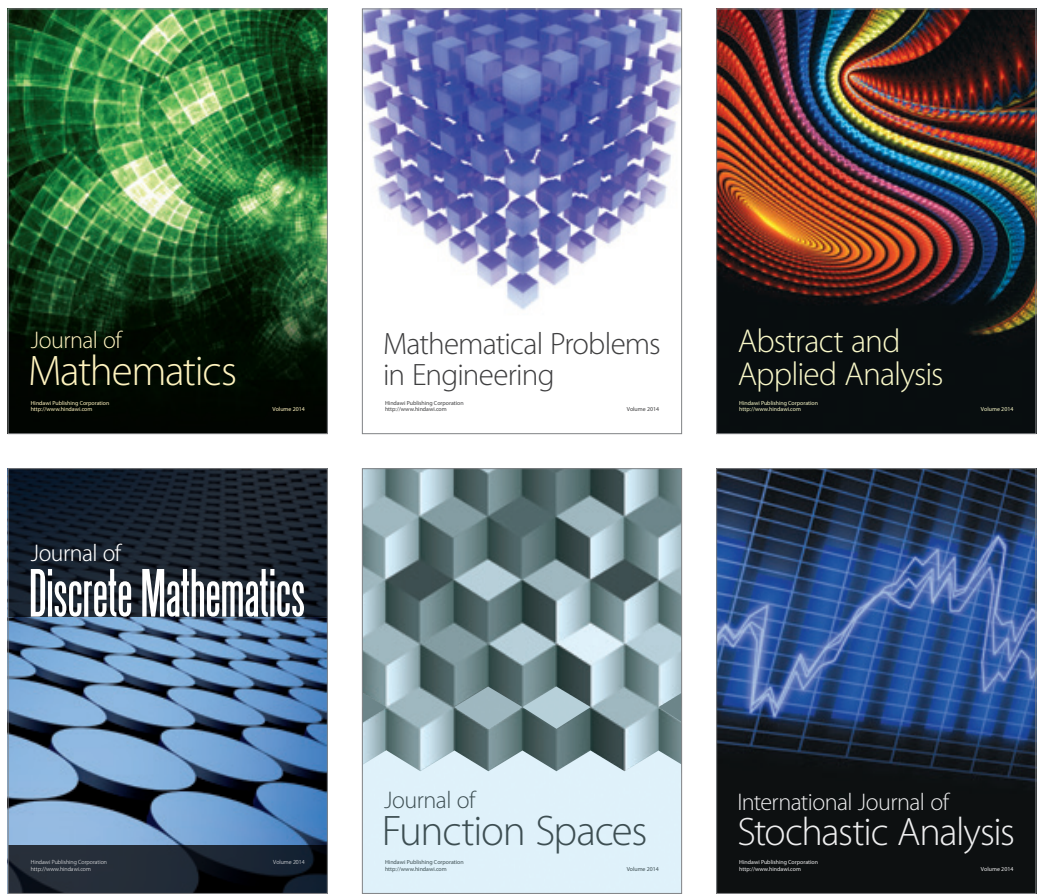

Journal of

Function Spaces

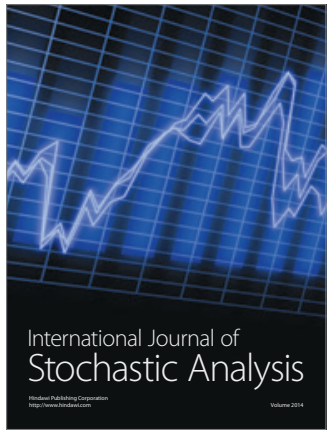

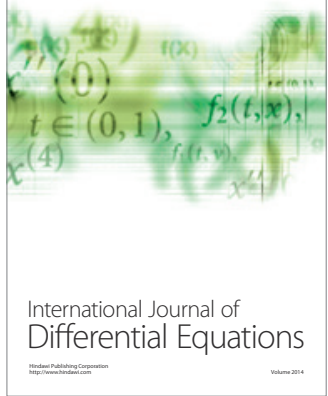
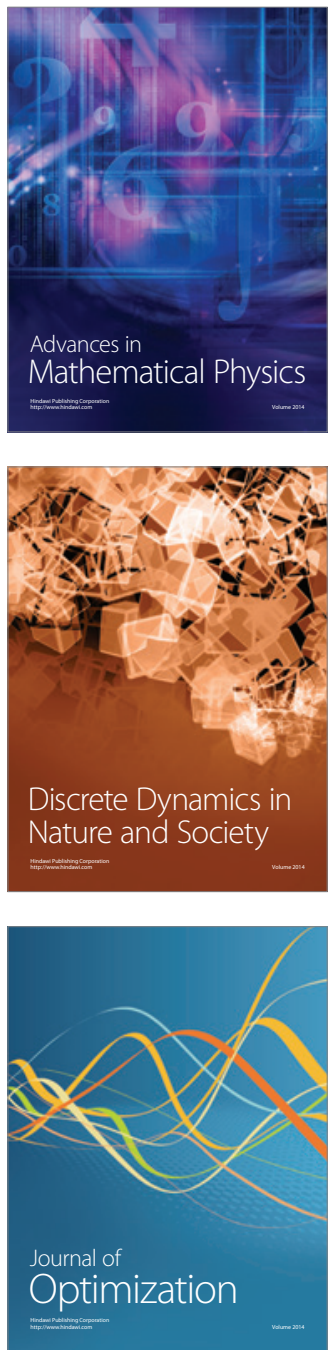